\title{
Finite subgraphs of uncountably chromatic graphs
}

\author{
Péter Komjáth* \\ Saharon Shelah ${ }^{\dagger}$
}

October 30,2018

\begin{abstract}
It is consistent that for every function $f: \omega \rightarrow \omega$ there is a graph with size and chromatic number $\aleph_{1}$ in which every $n$-chromatic subgraph contains at least $f(n)$ vertices $(n \geq 3)$. This solves a $\$ 250$ problem of Erdös. It is consistent that there is a graph $X$ with $\operatorname{Chr}(X)=|X|=\aleph_{1}$ such that if $Y$ is a graph all whose finite subgraphs occur in $X$ then $\operatorname{Chr}(Y) \leq \aleph_{2}$ (so the Taylor conjecture may fail). It is also consistent that if $X$ is a graph with chromatic number at least $\aleph_{2}$ then for every cardinal $\lambda$ there exists a graph $Y$ with $\operatorname{Chr}(Y) \geq \lambda$ all whose finite subgraphs are induced subgraphs of $X$.
\end{abstract}

\section{Introduction}

In [8] Erdős and Hajnal determined those finite graphs which appear as subgraphs in every uncountably chromatic graph: the bipartite graphs. In fact, not just that any odd circuit can be omitted, for every natural number $n \geq 1$ and infinite cardinal $\kappa$ there is a graph with cardinality and chromatic number $\kappa$ such that it omits all odd circuits up to length $2 n+1$. They observed that the so-called $r$-shift graph construction has all but one of these properties; the vertex set of $\operatorname{Sh}_{r}(\kappa)$ is the set of all $r$-tuples from $\kappa$, with $\left\{x_{0}, x_{1}, \ldots, x_{r-1}\right\}<$ joined to $\left\{x_{1}, x_{2}, \ldots, x_{r}\right\}_{<}$, then this graph omits odd circuits of length $3,5, \ldots, 2 r+1$ and the Erdös-Rado theorem asserts that the chromatic number of $\operatorname{Sh}_{r}\left(\exp _{r-1}(\kappa)^{+}\right)$ is at least $\kappa^{+}$.

The problem of determining the classes of finite graphs that occur in uncountably chromatic graphs seems to be much harder, and its investigation was strongly pushed by Erdős and Hajnal.

An early conjecture for example was the following. Every uncountably chromatic graph contains all odd circuits from some length onward. This was then

* Research partially supported by Hungarian National Research Grant T 032455.

${ }^{\dagger}$ This research was supported by the Israel Science Foundation. Publication 788. 
proved by Erdős, Hajnal, and Shelah [11], and independently, by Thomassen [17].

In [19] and later in [11] the following problem was posed (the Taylor conjecture). If $\kappa, \lambda$ are uncountable cardinals and $X$ is a $\kappa$-chromatic graph, is there a $\lambda$-chromatic graph $Y$ such that every finite subgraph of $Y$ appears as a subgraph of $X$. Notice that the above shift graphs give some evidence for this conjecture - the finite subgraphs of $\mathrm{Sh}_{r}(\kappa)$ do not depend on the parameter $\kappa$. The authors of [11] remarked that even the following much stronger conjecture seemed possible. If $X$ is uncountably chromatic, then for some $r$ it contains all finite subgraphs of $\operatorname{Sh}_{r}(\omega)$. This conjecture was then disproved in [13].

One easy remark, the so called Hanf number argument gives that there is a cardinal $\kappa$ with the property that if the chromatic number of some graph $X$ is at least $\kappa$ then there are arbitrarily large chromatic graphs with all finite subgraphs appearing in $X$. This argument, however, does not give any reasonable bound on $\kappa$.

Another conjecture of Erdős and Hajnal if the maximal chromatic number of $n$-element subgraphs of an uncountably chromatic graph as a function of $n$ can converge to infinity arbitarily slowly as $n$ tends to infinity. It was mentioned in several problem papers, for example in [2], [5], [6], [10], [12]. See also [1], [14]. The relevance of the above examples is that the chromatic number of the $n$-vertex subgraphs of (any) $\mathrm{Sh}_{r}(\kappa)$ grows roughly as the $r-1$ times iterated logarithm of $n$. Perhaps it was this fact that led Erdős and Hajnal to the above problem.

Erdös also tirelessly popularized the Taylor conjecture, he mentioned it e.g., in [3], [4], [7], [9], [10], [11]. It is also mentioned in [1], the book collecting Erdős' conjectures on graphs. In [15] we gave some results when the additional hypotheses $|X|=\kappa,|Y|=\lambda$ was imposed. We described countably many different classes $\mathcal{K}_{n, e}$ of finite graphs and proved that if $\lambda^{\aleph_{0}}=\lambda$ then every $\lambda^{+}$chromatic graph of cardinal $\lambda^{+}$contains, for some $n, e$, all elements of $\mathcal{K}_{n, e}$ as subgraphs. On the other hand, it is consistent for every regular infinite cardinal $\kappa$ that there is a $\kappa^{+}$-chromatic graph on $\kappa^{+}$that contains finite subgraphs only from $\mathcal{K}_{n, e}$. We got, therefore, some models of set theory, where the finite subraphs of graphs with $|X|=\operatorname{Chr}(X)=\kappa^{+}$for regular uncountable cardinals $\kappa$ were completely described.

Notice that the class of regular cardinals on which the above result operated excludes $\omega_{1}$, and in this paper we show the reason, by resolving the above Erdős-Hajnal conjecture: it is consistent that for every monotonically increasing function $f: \omega \rightarrow \omega$ there is a graph with size and chromatic number $\aleph_{1}$ in which every $n$-chromatic subgraph has at least $f(n)$ elements $(n \geq 3)$. The possibility of transforming the proof into a ZFC argument will be checked in the forthcoming [16], Chapter 9. An application of the method presented here gives the consistent existence of a graph $X$ with $\operatorname{Chr}(X)=|X|=\aleph_{1}$ such that if $Y$ is a graph (of any size) all whose subgraphs are subgraphs of $X$ then $\operatorname{Chr}(Y) \leq \aleph_{2}$. This gives a consistent negative answer to the Taylor conjecture. As for the positive direction we prove that it is consistent that if $X$ is a graph with chromatic number at least $\aleph_{2}$ then there are arbitrarily large chromatic 
graphs all whose finite subgraphs being induced subgraphs of $X$.

Theorems 1 and 2 were proved by S. Shelah and then P. Komjáth proved Theorems 3 and 4 .

Notation. We use the standard axiomatic set theory notation. If $A$ is a set of ordinals, $\beta$ is an ordinal, then $\beta<A$, means that $\beta<\alpha$ holds for every $\alpha \in A$. Similarly for $\beta \leq A, A<\beta$, etc. If $f$ is a function, $A$ a set, then we let $f[A]=\{f(x): x \in A\}$. If $S$ is a set, $\kappa$ is a cardinal, $[S]^{\kappa}=\{X \subseteq S:|X|=\kappa\}$, $[S]^{<\kappa}=\{X \subseteq S:|X|<\kappa\}$. A graph is an ordered pair $(V, X)$ where $V$ is some set (the set of vertices) and $X \subseteq[V]^{2}$ (the set of edges). In some cases we identify the graph and $X$. The chromatic number of a graph $(V, X)$ is the least cardinal $\mu$ such that there exists a function $f: V \rightarrow \mu$ with $f(x) \neq f(y)$ for $\{x, y\} \in X$ (a good coloring). A path of length $n$ is a sequence $\left\{v_{0}, \ldots, v_{n}\right\}$ of distinct vertices such that $\left\{v_{i}, v_{i+1}\right\} \in X$ holds for $i<n$. A cycle of length $n$ is a sequence $\left\{v_{1}, \ldots, v_{n}\right\}$ of vertices such that for $1 \leq i<n$ we have $\left\{v_{i}, v_{i+1}\right\} \in X$ and $\left\{v_{n}, v_{1}\right\} \in X$ also holds. If the vertices are distinct then we call it a circuit.

\section{A large chromatic graph with small chromatic finite subgraphs}

Let $f: \omega \rightarrow \omega$ be a strictly increasing function. Fix a sequence $\left\{C_{\alpha}: \alpha<\right.$ $\omega_{1}$, limit $\}$ such that $C_{\alpha}$ is an $\omega$-sequence converging to $\alpha$, and the whole sequence is a club guessing sequence, that is, if $C \subseteq \omega_{1}$ is a closed unbounded set, then $C_{\alpha} \subseteq C$ holds for some $\alpha$. Notice that the existence of a guessing sequence is an easy consequence of the diamond principle.

We are going to define the notion of forcing $\left(Q^{f}, \leq\right)$. Every condition $p \in Q^{f}$ will be of the form $p=\left(s, u, g, m, h_{i}, c\right)$ where $s \in\left[\omega_{1}\right]^{<\omega}, u \subseteq s$, consisting of limit ordinals, $g$ is a graph on $u$, for every $\alpha \in u$ we have an $m(\alpha)<\omega$, and then the ordinals $h_{0}(\alpha)<\cdots<h_{m(\alpha)-1}(\alpha)<\alpha$ which are $C_{\alpha}$-separated, that is, $\min \left(C_{\alpha}\right)<h_{0}(\alpha)$ and between $h_{i}(\alpha)$ and $h_{i+1}(\alpha)$ there is an element of $C_{\alpha}$ $\left(h_{i}(\alpha)\right.$ is undefined if $i \geq m(\alpha)$ or $\left.\alpha \notin u\right) . c: g \rightarrow \omega$ satisfies that if $\{x, \alpha\} \in g$, $\{y, \alpha\} \in g$ and $x, y<\alpha$ then $c(x, \alpha) \neq c(y, \alpha)$.

Given $p \in Q^{f}$ we define

$$
Y_{r}^{p}=\{e \in g: c(e) \geq r\}
$$

for any natural number $r$.

We add the following stipulations.

(1.) If $\alpha \in u$ is incident to some $e \in g$ with $c(e) \geq r$ then $m(\alpha) \geq r$.

(2.) If $x, y$ are connected in $Y_{r}^{p}$ in $i$ steps then $h_{j-i}(x)<h_{j}(y)<h_{j+i}(x)$ holds for $i \leq j \leq f(r)-i$.

(3.) $Y_{r}^{p}$ does not contain odd circuits of length $\leq f(r)$. 
We notice that although a condition $p=\left(s, u, g, m, h_{i}, c\right)$ is infinite (perhaps $p=\left(s, u, g, m,\left(h_{i}\right)_{i<\omega}, c\right)$ or $p=\left(s, u, g, m, h_{i}, c\right)_{i<\omega}$ would be better notation) as all but finitely many of the partial functions $\left\{h_{i}: i<\omega\right\}$ are the empty function, every condition is really a finite object.

The partial order on $Q^{f}$ is defined the natural way. $p^{\prime}=\left(s^{\prime}, u^{\prime}, g^{\prime}, m^{\prime}, h_{i}^{\prime}, c^{\prime}\right)$ extends $p=\left(s, u, g, m, h_{i}, c\right)$ iff the following hold. $s^{\prime} \supseteq s, u=u^{\prime} \cap s, g=g^{\prime} \cap[s]^{2}$. $m^{\prime}(\alpha) \geq m(\alpha)$ holds for $\alpha \in u$, and $h_{i}^{\prime}(\alpha)=h_{i}(\alpha)$ for $i<m(\alpha)$. Finally, $c^{\prime}(x, \alpha)=c(x, \alpha)$ holds for $\{x, \alpha\} \in g$.

We call two conditions $p=\left(s, u, g, m, h_{i}, c\right)$ and $p^{\prime}=\left(s^{\prime}, u^{\prime}, g^{\prime}, m^{\prime}, h_{i}^{\prime}, c^{\prime}\right)$ isomorphic iff $|s|=\left|s^{\prime}\right|$ and the unique order preserving mapping $\pi: s \rightarrow s^{\prime}$ satisfies $u^{\prime}=\pi[u], g^{\prime}=\pi[g], m(\alpha)=m^{\prime}(\pi(\alpha))$ for $\alpha \in u, h_{i}^{\prime}(\pi(\alpha))=h_{i}(\alpha)$ and $c^{\prime}(\pi(x), \pi(\alpha))=c(x, \alpha)$ hold whenever the right hand sides are defined. Notice that, as our conditions are finite structures, we have only countably many isomorphism types.

From a generic $G \subseteq Q^{f}$ we define the following graphs on $\omega_{1}$ :

$$
\begin{gathered}
X=\bigcup\left\{g:\left(s, u, g, m, h_{i}, c\right) \in G\right\}, \\
X_{n}=\left\{\{x, y\}:\{x, y\} \in g, c(x, y)=n,\left(s, u, g, m, h_{i}, c\right) \in G\right\}, \\
Y_{r}=X_{r} \cup X_{r+1} \cdots
\end{gathered}
$$

and notice that $Y_{r}=\cup\left\{Y_{r}^{p}: p \in G\right\}$.

Lemma 1. For $\alpha<\omega_{1}$ the set $\left\{\left(s, u, g, m, h_{i}, c\right) \in Q^{f}: \alpha \in s\right\}$ is dense.

Proof. Straightforward.

Lemma 2. If $\alpha<\omega_{1}$ is limit, $\left(s, u, g, m, h_{i}, c\right) \in Q^{f}, \alpha \notin s$, then there is an extension $\left(s^{\prime}, u^{\prime}, g^{\prime}, m^{\prime}, h_{i}^{\prime}, c^{\prime}\right) \leq\left(s, u, g, m, h_{i}, c\right)$ with $\alpha \in u^{\prime}$.

Proof. Straightforward.

Lemma 3. If $\left(s, u, g, m, h_{i}, c\right) \in Q^{f}, \alpha \in u, n<\omega$ then there is an extension $\left(s^{\prime}, u^{\prime}, g^{\prime}, m^{\prime}, h_{i}^{\prime}, c^{\prime}\right) \leq\left(s, u, g, m, h_{i}, c\right)$ with $m(\alpha) \geq n$.

Proof. It suffices to show that $m(\alpha)$ can be incremented by one. Given $h_{m(\alpha)-1}(\alpha)<\alpha$ if we choose $h_{m(\alpha)}^{\prime}(\alpha)<\alpha$ large enough the condition on $C_{\alpha}$ will surely be satisfied.

Lemma 4. $\left(Q^{f}, \leq\right)$ is ccc.

Proof. Modulo standard arguments we have to show that $p=\left(s, u, g, m, h_{i}, c\right)$ and $p^{\prime}=\left(s^{\prime}, u^{\prime}, g^{\prime}, m^{\prime}, h_{i}^{\prime}, c^{\prime}\right)$ are compatible, assuming that they are isomorphic, and $s \cap s^{\prime}$ is an initial segment of both $s$ and $s^{\prime}$. Let $\pi: s \rightarrow s^{\prime}$ be the order preserving structure isomorphism. We let $p^{\prime \prime}=\left(s^{\prime \prime}, u^{\prime \prime}, g^{\prime \prime}, m^{\prime \prime}, h_{i}^{\prime \prime}, c^{\prime \prime}\right)$ where we take unions in all coordinates.

In order to show that $p^{\prime \prime}$ is a condition we have to check properties (1.-3.).

(1.) is obvious. 
For (3.) assume that $C$ is an odd circuit of length $\leq f(r)$ in $Y_{r}^{p^{\prime \prime}}$. If we replace every $e \in C$ that contains at least one vertex from $s-s^{\prime}$ with $\pi(e)$ then we get an odd cycle $C^{\prime}$ in $Y_{r}^{p^{\prime}}$. $C^{\prime}$ splits into circuits, at least one of them odd, so we get a contradiction.

For (2.) notice that it holds if $x, y$ are joined via a path going entirely in $Y_{r}^{p}$ or $Y_{r}^{p^{\prime}}$. It suffices, therefore, to show that if some path $P$ between $x$ and $y$ of length $i$ is split by an inner point $z$ into the paths $P_{0}$ and $P_{1}$ between $x$ and $z$, and $z$ and $y$, respectively, and of the respective lengths $i_{0}$ and $i_{1}$ (so $i_{0}+i_{1}=i$ ) and the statement holds for $P_{0}$ and $P_{1}$ then it holds for $P$, as well. Indeed, for $i \leq j \leq f(r)-i$ we have

$$
h_{j-\left(i_{0}+i_{1}\right)}(x)<h_{j-i_{1}}(z)<h_{j}(y)<h_{j+i_{1}}(z)<h_{j+\left(i_{0}+i_{1}\right)}(x) .
$$

Lemma 5. $\operatorname{Chr}(X)=\omega_{1}$.

Proof. Assume that some $\bar{p}$ forces that $\underline{F}$ is a good coloring of $X$ with the elements of $\omega$. Select an increasing, continuous sequence of countable elementary submodels $\bar{p}, \underline{F}, Q^{f}, \|-\in N_{0} \prec N_{1} \prec \cdots N_{\alpha} \prec H(\lambda)$ with some large enough regular cardinal $\lambda$, for $\alpha<\omega_{1}$, such that $\gamma_{\alpha}=N_{\alpha} \cap \omega_{1}$ is an ordinal. The set $C=\left\{\gamma_{\alpha}: \alpha<\omega_{1}\right\}$ will be a closed, unbounded set, so by the guessing property there is some $\delta=\gamma_{\alpha}$ such that $C_{\delta} \subseteq C$. Notice that all points of $\bar{p}$ are smaller than $\delta$.

Extend $\bar{p}$ to a $p^{\prime}$ using Lemma 2., adding $\delta$ to the $u$-part, then let $p^{*}$ be a condition extending $p^{\prime}$ such that $p^{*} \|-\underline{F}(\delta)=i$ holds for some $i<\omega$.

Let $n$ be some natural number that $n \notin\left\{c^{*}(x, \delta):\{x, \delta\} \in g^{*}, x<\delta\right\}$ where $p^{*}=\left(s^{*}, u^{*}, g^{*}, m^{*}, h_{i}^{*}, c^{*}\right)$. Extend $p^{*}$ using Lemma 3., to some condition $p$ with $p=\left(s, u, g, m, h_{i}, c\right)$ such that $m=m(\delta) \geq f(n)$ holds.

In $p$ we have the values

$$
h_{0}(\delta)<h_{1}(\delta)<\cdots<h_{m-1}(\delta)<\delta
$$

and by our requirements on conditions there are elements $\delta_{0}, \ldots, \delta_{m-1}$ of $C_{\delta}$ such that

$$
\delta_{0}<h_{0}(\delta)<\delta_{1}<h_{1}(\delta)<\cdots<\delta_{m-1}<h_{m-1}(\delta)<\delta
$$

holds.

The values $\delta_{0}, \ldots, \delta_{m-1}, \delta$ break $s$ into disjoint parts: $s=s_{0} \cup \cdots \cup s_{m} \cup s_{m+1}$ with

$$
s_{0}<\delta_{0} \leq s_{1}<\delta_{1} \leq s_{2}<\cdots<\delta_{m-1} \leq s_{m}<\delta \leq s_{m+1}
$$

(some of them may be empty).

Sublemma. There is a condition $p^{\prime}$ on some $s^{\prime}=s_{0} \cup s_{1}^{\prime} \cup \cdots \cup s_{m}^{\prime} \cup s_{m+1}^{\prime}$ isomorphic to $p$ with $\left|s_{i}^{\prime}\right|=\left|s_{i}\right|, \delta^{\prime}=\min \left(s_{m+1}^{\prime}\right), p^{\prime} \|-\underline{F}\left(\delta^{\prime}\right)=i$ and

$s_{0}<s_{1}^{\prime}<\delta_{0} \leq s_{1}<s_{2}^{\prime}<\delta_{1} \leq s_{2}<\cdots<s_{m}^{\prime}<\delta_{m-1} \leq s_{m}<s_{m+1}^{\prime}<\delta \leq s_{m+1}$ 
holds.

Proof. Let $\theta$ be the isomorphism type of $p$. For the ordered finite sets $\bar{x}_{0}, \bar{x}_{1}, \ldots, \bar{x}_{m+1}$ let $\psi\left(\bar{x}_{0}, \bar{x}_{1}, \ldots, \bar{x}_{m+1}\right)$ denote the statement that $\bar{x}_{0}<\bar{x}_{1}<$ $\cdots<\bar{x}_{m+1},\left|\bar{x}_{i}\right|=\left|s_{i}\right|$ and for the (unique) condition $p$ on $\bar{x}_{0} \cup \bar{x}_{1} \cup \cdots \cup \bar{x}_{m+1}$ of type $\theta p \|-\underline{F}(\delta)=i$ where $\delta=\min \left(\bar{x}_{m+1}\right)$.

Set

$$
\varphi_{m+1}\left(\bar{x}_{0}, \bar{x}_{1}, \ldots, \bar{x}_{m+1}\right)=\psi\left(\bar{x}_{0}, \bar{x}_{1}, \ldots, \bar{x}_{m+1}\right)
$$

and for $0 \leq i \leq m$

$$
\varphi_{i}\left(\bar{x}_{0}, \bar{x}_{1}, \ldots, \bar{x}_{i}\right)=\exists^{*} \bar{x}_{i+1} \varphi_{i+1}\left(\bar{x}_{0}, \bar{x}_{1}, \ldots, \bar{x}_{i+1}\right)
$$

where the quantifier $\exists^{*}$ denotes "there exist unboundedly many" which is expressible in the first order language of $\left(\omega_{1},<\right)$.

Claim. For $0 \leq i \leq m+1$ the sentence $\varphi_{i}\left(s_{0}, s_{1}, \ldots, s_{i}\right)$ holds.

Proof. We prove the statement by reverse induction on $0 \leq i \leq m+1$. We certainly have $\varphi_{m+1}\left(s_{0}, s_{1}, \ldots, s_{m+1}\right)$. If for some $0 \leq i \leq m$ we had that $\varphi_{i+1}\left(s_{0}, s_{1}, \ldots, s_{i+1}\right)$ holds yet

$$
\varphi_{i}\left(s_{0}, s_{1}, \ldots, s_{i}\right)=\exists^{*} \bar{x}_{i+1} \varphi_{i+1}\left(s_{0}, s_{1}, \ldots, s_{i}, \bar{x}_{i+1}\right)
$$

fails, then there was a bound, computable from $s_{0}, s_{1}, \ldots, s_{i}$ for the minima of those sets $\bar{x}_{i+1}$ for which $\varphi_{i+1}\left(s_{0}, s_{1}, \ldots, s_{i}, \bar{x}_{i+1}\right)$ holds. Then this bound was smaller than $\delta_{i}$ ( $\delta$ for $i=m$ ) as there is an elementary submodel containing the ordinals $<\delta_{i}($ or $<\delta)$ but this contradicts the fact that $\varphi_{i+1}\left(s_{0}, s_{1}, \ldots, s_{i+1}\right)$ holds and $\delta_{i} \leq s_{i}$.

Using the Claim we can inductively select the sets $s_{1}^{\prime}, \ldots, s_{m+1}^{\prime}$ such that for every $1 \leq i \leq m+1$ we have $\varphi_{i}\left(s_{0}, s_{1}^{\prime}, \ldots, s_{i}^{\prime}\right)$ and $s_{i}<s_{i+1}^{\prime} \leq \delta_{i}$, as required.

Using the Sublemma we create the following one-edge amalgamation $p^{\prime \prime}$ of $p$ and $p^{\prime} . p^{\prime \prime}=\left(s^{\prime \prime}, u^{\prime \prime}, g^{\prime \prime}, m^{\prime \prime}, h_{i}^{\prime \prime}, c^{\prime \prime}\right)$ where $s^{\prime \prime}=s \cup s^{\prime}, u^{\prime \prime}=u \cup u^{\prime}, g^{\prime \prime}=$ $g \cup g^{\prime} \cup\left\{\left\{\delta, \delta^{\prime}\right\}\right\}, m^{\prime \prime}=m \cup m^{\prime}, h_{i}^{\prime \prime}=h_{i} \cup h_{i}^{\prime}, c^{\prime \prime}=c \cup c^{\prime}$.

We have to show that $p^{\prime \prime}$ is indeed a condition, that is, we have to check if the properties (1.-3.) hold.

(1.) is obvious.

For (2.) we argue as in the proof of Lemma 4; every path in question is the union of paths for which this condition holds, plus possibly the path $\left\{\delta, \delta^{\prime}\right\}$ but (2.) also holds for this.

Assume finally, that $C$ is a circuit of length $2 t+1 \leq f(r)$ in $Y_{r}^{p^{\prime \prime}}$. Unless $C$ contains $\left\{\delta, \delta^{\prime}\right\}$, we can argue as in Lemma 4 . So we are left with the case that $C$ contains $\left\{\delta, \delta^{\prime}\right\}$ and $r \leq n$. That is, $\delta \in s$ and $\delta^{\prime} \in s^{\prime}$ are joined in $Y_{r}^{p} \cup Y_{r}^{p^{\prime}}$ in $2 t$ steps, and this is only possible if the connecting path has vertices in $s \cap s^{\prime}$. So we get that $\delta$ can be connected in $Y_{r}^{p}$ with some point in $s \cap s^{\prime}$ in $\leq t$ steps. But this is impossible: if $x \in s \cap s^{\prime}$ is such a point then $h_{0}(\delta)<h_{t}(x)<h_{2 t}(\delta)$ by condition (2.) and also $x<h_{0}(\delta)$, a contradiction. 
As $p^{\prime \prime}$ forces that $F(\delta)=F\left(\delta^{\prime}\right)=i$ yet they are joined in $X$, we are finished.

Theorem 1. The forcing $Q^{f}$ adds an uncountably chromatic graph $X$ on $\omega_{1}$ such that every subgraph on at most $f(r)$ vertices is at most $2^{r+1}$-chromatic.

Proof. As every $X_{n}$ is a circuitfree graph, it can be colored with two colors. Consider now a subgraph of $X$ induced by a set $S$ of at most $f(r)$ vertices. On $S$ all the graphs $X_{0}, \ldots, X_{r-1}$ are bipartite, and so is $Y_{r}=X_{r} \cup \cdots$ (as it has no odd circuits of length $\leq f(r)$ ). So their union, $X$ restricted to $S$, can be colored by at most $2^{r+1}$ colors.

Theorem 2. It is consistent with $C H$ that for every function $f: \omega \rightarrow \omega$ there is an uncountably chromatic graph $X$ on $\omega_{1}$ such that every sugraph of $X$ on $f(r)$ vertices is at most $r$-chromatic $(r \geq 2)$.

Proof. Assume that $\diamond$ holds in the ground model. Then we have $\mathrm{CH}$ and there is a club guessing sequence $\left\{C_{\alpha}: \alpha<\omega_{1}\right.$, limit $\}$ as required for Theorem 1 . We force with a finite support iteration $P=\left\{P_{\alpha}, Q_{\alpha}: \alpha<\omega_{1}\right\}$. In step $\alpha<\omega_{1}$ we add $Q_{\alpha}=Q^{f_{\alpha}}$ for some increasing function $f_{\alpha}: \omega \rightarrow \omega$. As this will be a ccc forcing that preserves $\mathrm{CH}$ it is possible by bookkeeping to make sure that every suitable $f: \omega \rightarrow \omega$ occurs as some $f_{\alpha}$. Also, as $P_{\alpha}$, the iteration up to $\alpha$ is ccc, every closed, unbounded set $C$ in $V^{P_{\alpha}}$ contains a ground model closed, unbounded set $D$, and as there is some element $C_{\alpha}$ of the club guessing system that $C_{\alpha} \subseteq D$ we have $C_{\alpha} \subseteq C$, that is the club guessing system retains its property in $V^{P_{\alpha}}$.

Call a condition $p \in P$ determined if for every $\alpha<\omega_{1}$ the condition $p \mid \alpha$ completely determines $p(\alpha)$, that is, for every $\alpha$ coordinate $p(\alpha)$ is not just a name for a finite structure but it is actually a finite structure.

Lemma 6. The determined conditions form a dense set in $P$.

Proof. We prove by induction on $\alpha<\omega_{1}$ that the determined conditions form a dense subset of $P_{\alpha}$. This is obvious if $\alpha$ is limit, as we are considering finite supports. Assume that we have the statement for some $\alpha<\omega_{1}$ and we try to handle the case of $\alpha+1$. Let $(p, q) \in P_{\alpha+1}=P_{\alpha} * Q_{\alpha}$ be arbitrary. Extend $p$ to some $p^{\prime}$ that completely determines $q$, that is, there is a finite structure $h$ that $p^{\prime} \|-q=h$. Then extend $p^{\prime}$ to a determined $p^{*} \in P_{\alpha}$. Now $\left(p^{*}, h\right)$ is a determined extension of $(p, q)$.

Lemma 7. For every $\alpha<\omega_{1}, \operatorname{Chr}\left(X_{\alpha}\right)=\omega_{1}$ holds in $V^{P}$.

Proof. By moving to $V^{P_{\alpha}}$ we can assume that $\alpha=0$. We imitate the proof of Lemma 5. By Lemma 6 we can work with determined conditions. We consider every such condition as a finite structure on some finite subset $s$ of $\omega_{1}$, here $s$ contains all points of all graphs $p(\beta)$ where $\beta$ is an arbitrary element of the support of $p$, and we also add the elements of the support to $s$. Assume that some $\bar{p} \in P$ forces that $\underline{F}$ is a good coloring of $X_{0}$ with the elements of $\omega$. With an argument like in Lemma 5 we get some natural number $n$, ordinals $\delta^{\prime}<\delta<$ $\omega_{1}$, and also $\alpha_{1}, \ldots, \alpha_{m}, \beta_{1}, \ldots, \beta_{t}, \beta_{1}^{\prime}, \ldots, \beta_{t}^{\prime}$ and two isomorphic determined 
conditions $p$ and $p^{\prime}$ with the respective supports $\left\{0, \alpha_{1}, \ldots, \alpha_{m}, \beta_{1}, \ldots, \beta_{t}\right\}$ and $\left\{0, \alpha_{1}, \ldots, \alpha_{m}, \beta_{1}^{\prime}, \ldots, \beta_{t}^{\prime}\right\}$ that $p\left\|-\underline{F}(\delta)=n, p^{\prime}\right\|-\underline{F}\left(\delta^{\prime}\right)=n$ hold, $p(0)$ and $p^{\prime}(0)$ are isomorphic conditions that behave like $p$ and $p^{\prime}$ in Lemma 5 , for every $\alpha_{i}$ the structures $p\left(\alpha_{i}\right)$ and $p^{\prime}\left(\alpha_{i}\right)$ are isomorphic with the common part preceding the tails, that is, it is possible to make a non-edge amalgamation. We can, therefore, take the union of $p$ and $p^{\prime}$, and add the edge $\left\{\delta, \delta^{\prime}\right\}$ with color $n$ in coordinate 0 .

The above Lemma concludes the proof of the Theorem.

\section{The Taylor conjecture}

Theorem 3. It is consistent that there is a graph $X$ with $\operatorname{Chr}(X)=|X|=\aleph_{1}$ such that if $Y$ is a graph with all finite subgraphs occurring in $X$ then $\operatorname{Chr}(Y) \leq$ $\aleph_{2}$, that is, the Taylor conjecture fails.

Proof. Let $V$ be a model of GCH and $\diamond$. Let $P$ be the notion of forcing that adds a Cohen real. It is well known that $P$ adds an undominated real, that is, a function $f: \omega \rightarrow \omega$ such that for no $g: \omega \rightarrow \omega$ in $V$ does $f(n) \leq g(n)$ hold for every $n<\omega$. Let $V^{\prime}$ be the forced model. Notice that $V^{\prime}$ still has GCH and club guessing (by the argument in the proof of Theorem 2.). Now force over $V^{\prime}$ with the partial order $Q^{f}$, and get a graph $X$ with $\operatorname{Chr}(X)=|X|=\aleph_{1}$ such that every $n$-chromatic subgraph of $X$ has at least $f(n)$ elements $(n \geq 3)$. This $X$ will be our graph. To show the property stated, assume that $Y$ is a graph in $V^{P, Q^{f}}$ whose every finite subgraph is a subgraph of $X$. We assume that the vertex set of $Y$ is some cardinal $\lambda$. We notice that every $n$-chromatic subgraph of $Y$ has at least $f(n)$ elements.

Lemma 8. If $Z \subseteq Y$ is a subgraph with $Z \in V$ then $Z$ is finitely chromatic.

Proof. Otherwise for every $n<\omega$ we can let $g(n)$ be the minimal size of an $n$-chromatic subgraph of $Z$. Now notice that $g \in V$ and also by the absoluteness of the set of finite subsets of $\lambda$ and the absoluteness of the cromatic number of a finite graph, $g$ denotes the same thing in $V$ and $V^{P * Q^{f}}$. This implies that $g(n) \geq f(n)$ holds for every $n$, but that obviously contradicts the fact that $f$ cannot be dominated by the the ground model $\omega \rightarrow \omega$ functions.

We finally need the following Lemma.

Lemma 9. If $R$ is a notion of forcing over some model $V, Y$ is a graph in the extended model on some ordinal $\lambda$ then $Y$ is the union of at most $|R|$ subgraphs which are elements of $V$.

Proof. Let $\tau$ be a name for (the edge set of) $Y$. Set

$$
Z_{p}=\left\{e \in[\lambda]^{2}: p \|-e \in \tau\right\}
$$

for $p \in R$, then $Y=\bigcup\left\{Z_{p}: p \in G\right\}$ where $G \subseteq R$ is a generic set. 
To finish the proof of the Theorem we remark that by Lemma $9 Y$ decomposes into the union of $\left|P * Q^{f}\right|=\aleph_{1}$ graphs each being in $V$, therefore finitely chromatic, so we get $\operatorname{Chr}(Y) \leq 2^{\aleph_{1}}=\aleph_{2}$.

The followig argument gives that the Hanf number mentioned in the Introduction can be as small as $\aleph_{2}$.

Theorem 4. It is consistent that if $X$ is a graph with $\operatorname{Chr}(X) \geq \aleph_{2}$ then for every cardinal $\lambda$ there exists a graph $Y$ with $\operatorname{Chr}(Y) \geq \lambda$ all whose finite subgraphs are induced subgraphs of $X$.

Proof. Let $V$ be a model of GCH. Choose the regular cardinal $\kappa$ so large that the following holds. If $X$ is a graph with $\operatorname{Chr}(X) \geq \kappa$ and $\lambda$ is a cardinal then there is a graph with $\operatorname{Chr}(Y) \geq \lambda$ all whose finite subgraphs occur as subgraphs of $X$. Clearly, such a $\kappa$ exists.

Let $P=\operatorname{Col}(\omega, \kappa)$ be the collapse of $\kappa$ to $\aleph_{0}$, that is, the elements of $P$ are those functions of the form $p: n \rightarrow \kappa$ for some $n<\omega$ with $p \leq q$ iff $p$ extends $q$ as a function. Our claim is that if $G \subseteq P$ is generic then $V[G]$ models the statement of the Theorem. Notice that $|\kappa|=\aleph_{0}$ holds there and calculation shows that GCH still holds in $V[G]$.

Assume that $X$ is a graph in $V[G]$ with chromatic number at least $\aleph_{2}$ (that is, $\aleph_{2}^{V[G]}$ ). By Lemma 9., $X$ is the union of $|G|=\aleph_{0}$ ground model graphs. As $\aleph_{1}^{\aleph_{0}}=\aleph_{1}$, one of them, say $Y$ must have chromatic number at least $\aleph_{2}$. In $V$, $Y$ has chromatic number at least $\aleph_{2}^{V[G]}=\kappa^{++}$. Assume that we are given some $\lambda>\kappa$. By the choice of $\kappa$, there is a graph $Z$ with $\operatorname{Chr}(Z) \geq \lambda$ such that every finite induced subgraph of $Z$ is an induced subgraph of $Y$.

Lemma 10. $\operatorname{Chr}(Z) \geq \lambda$ holds in $V[G]$.

Proof. Otherwise let $\underline{F}$ be a name for a coloring with the ordinals less than $\tau<\lambda$. Then the coloring $x \mapsto(p, \xi)$ is a good coloring of the vertices of $Z$ with $\kappa+\tau<\lambda$ colors, where $p \in P$ is some element of $P$ with $p \|-\underline{F}(x)=\xi$.

We are almost finished, the only problem is that the finite induced subgraphs of $Z$ are not induced subgraphs of $X$, they only are (edge-)subgraphs of induced subgraphs of $X$. The following Lemma is what we need.

Lemma 11. There is a graph $Z^{\prime}$ on the vertex set of $Z$ with $Z^{\prime} \supseteq Z$ and such that every induced subgraph of $Z^{\prime}$ is an induced subgraph of $X$.

Proof. Let $S$ be the vertex set of $Z$. For every finite subset $s$ of $S$ there are some graphs on $s$, which on the one hand are isomorphic to induced subgraphs of $X$, on the other hand they are supergraphs of the graph $Z$ restricted to $s$. Call these graphs appropriate for $s$. Notice that there are finitely many appropriate graphs for every given $s$, and if $T$ is an appropriate graph for $s$ and $s^{\prime}$ is a subset of $s$ then $T$ restricted to $s^{\prime}$ is a graph appropriate for $s^{\prime}$. We can therefore apply the Rado selection principle (or the compactness theorem of model theory) and get a graph $Z^{\prime}$ on $S$ every induced subgraph of which is appropriate, so $Z^{\prime}$ is a required.

As $\operatorname{Chr}\left(Z^{\prime}\right) \geq \operatorname{Chr}(Z) \geq \lambda$ holds we are done. 


\section{References}

[1] F. Chung, R. Graham: Erdős on graphs, A K Peters, 1998.

[2] P. Erdős: Problems and results on finite and infinite combinatorial analysis, Infinite and finite sets (Colloq. Keszthely 1973; dedicated to P. Erdös on his 60th birthday), Vol. I. Colloq. Math. Soc. J. Bolyai, Vol. 10, North Holland, Amsterdam, 1975, 403-424.

[3] P. Erdős: On the combinatorial problems which I would most like to see solved, Combinatorica 1 (1981), 25-42.

[4] P. Erdős: Problems and results on chromatic numbers in finite and infinite graphs, Graph theory with applications to algorithms and computer science (Kalamazoo, Mich. 1984), Wiley-Interscience Publ., Wiley, New York, 1985, 201-213,

[5] P. Erdős: Some of my favourite unsolved problems, A tribute to Paul Erdős, Cambridge Univ. Press, Cambridge, 1990., 467-478.

[6] P. Erdős: On some of my favourite problems in various branches of combinatorics, in: Proc. Fourth Czechoslovak Symp. on Combinatorics, Graphs and Complexity (Prachatice, 1990), Ann. Discrete Math. (eds.: J. Nešetřil and M. Fiedler), 51(1992), North-Holland, 69-79.

[7] P. Erdős: On some of my favourite theorems, Combinatorics. Paul Erdös is Eighty, II, (ed. D. Miklós, V. T. Sós, T. Szőnyi), 97-132.

[8] P.Erdős, A.Hajnal: On chromatic number of graphs and set-systems, Acta Math. Acad. Sci. Hung. 17 (1966), 61-99.

[9] P. Erdős, A. Hajnal: Unsolved and solved problems in set theory, Proceedings of the Tarski Symposium (Proc. Symp. Pure Math., Vol. XXV, Univ. of California, Berkeley, Calif., 1971), Amer.Math.Soc., Providence, R.I., 1974, 269-287.

[10] P. Erdős, A. Hajnal: Chromatic number of finite and infinite graphs and hypergraphs. (French summary) Special volume on ordered sets and their applications (L'Arbresle, 1982). Discrete Math. 53(1985), 281-285.

[11] P.Erdős, A.Hajnal, S.Shelah: On some general properties of chromatic number, in: Topics in Topology, Keszthely (Hungary), 1972, Coll. Math. Soc. J. Bolyai 8, 243-255.

[12] P. Erdős, A. Hajnal, E. Szemerédi: On almost bipartite large chromatic graphs, Annals of Discrete Math. 12(1982), 117-123.

[13] A. Hajnal, P.Komjáth: What must and what need not be contained in a graph of uncountable chromatic number? Combinatorica 4 (1984), 47-52. 
[14] T. R. Jensen, B. Toft: Graph coloring problems, John Wiley and Sons, 1995.

[15] P.Komjáth, S. Shelah: On Taylor's problem, Acta Math. Hung., 70 (1996), $217-225$.

[16] S. Shelah: Non-structure Theory, Oxford University Press, to appear.

[17] C. Thomassen: Cycles in graphs of uncountable chromatic number, Combinatorica 3 (1983), 133-134.

[18] W.Taylor: Atomic compactness and elementary equivalence, Fund. Math. 71 (1971), 103-112.

[19] W.Taylor: Problem 42, Comb. Structures and their applications, Proc. of the Calgary International Conference, 1969.

Péter Komjáth

Department of Computer Science

Eötvös University

Budapest, P.O.Box 120

1518, Hungary

e-mail: kope@cs.elte.hu
Saharon Shelah

Institute of Mathematics,

Hebrew University,

Givat Ram, 91904,

Jerusalem, Israel

e-mail: shelah@math.huji.ac.il 